Thorax (1949), 4, 168.

\title{
PULMONARY ASPERGILLOSIS IN COAL WORKERS
}

BY

\section{A. G. HEPPLESTON AND S. ROODHOUSE GLOYNE*}

From the Department of Pathology, Welsh National School of Medicine, Cardiff, and the Institute of Social Medicine, Oxford

Aspergillus infection of the lungs is known to affect particularly those individuals whose hobbies or occupations bring them into prolonged and intimate contact with domestic birds or grain (Jacobson, 1932 ; van Ordstrand, 1940 ; Coe, 1945). These are generally accepted as common sources of infection occurring by the respiratory route. The condition thus appears to have a distinct relationship to occupation. In an extensive search of the literature no instance has been discovered in which pulmonary aspergillosis occurred in a miner with or without pneumoconiosis, and there is no reference to it in the textbook on aspergilli by Thom and Raper (1945). The rarity with which dusty occupations and fungus infection are associated is attested by the fact that Case 1 represents the sole occasion on which aspergillosis or any other fungus disease has been detected in a continuous series of over 650 necropsies performed on Welsh coal workers during the past five years, and Case 2 is the only instance in a miscellaneous group of 267 necropsies on coal miners from various other coal fields in Great Britain.

*Working with a grant from the Medical Research Council.

FIG.1.-Thick section of right lung of Case 1 showing massive fibrosis with dust macules and their focal emphysema. Area of aspergillus infection at anterior margin of upper lobe indicated by the arrow. ( $\times \frac{1}{2}$ : unstained.)

\section{CAse Reports}

Case 1.-C.D.E., aged 56, had been a coal hewer at $\dot{\omega}$ several of the steam coal mines in South Wales for a $\vec{\sigma}$ total of $36 \frac{1}{2}$ years and had worked on the roadways underground for a further two years. During the last $\frac{\circ}{3}$

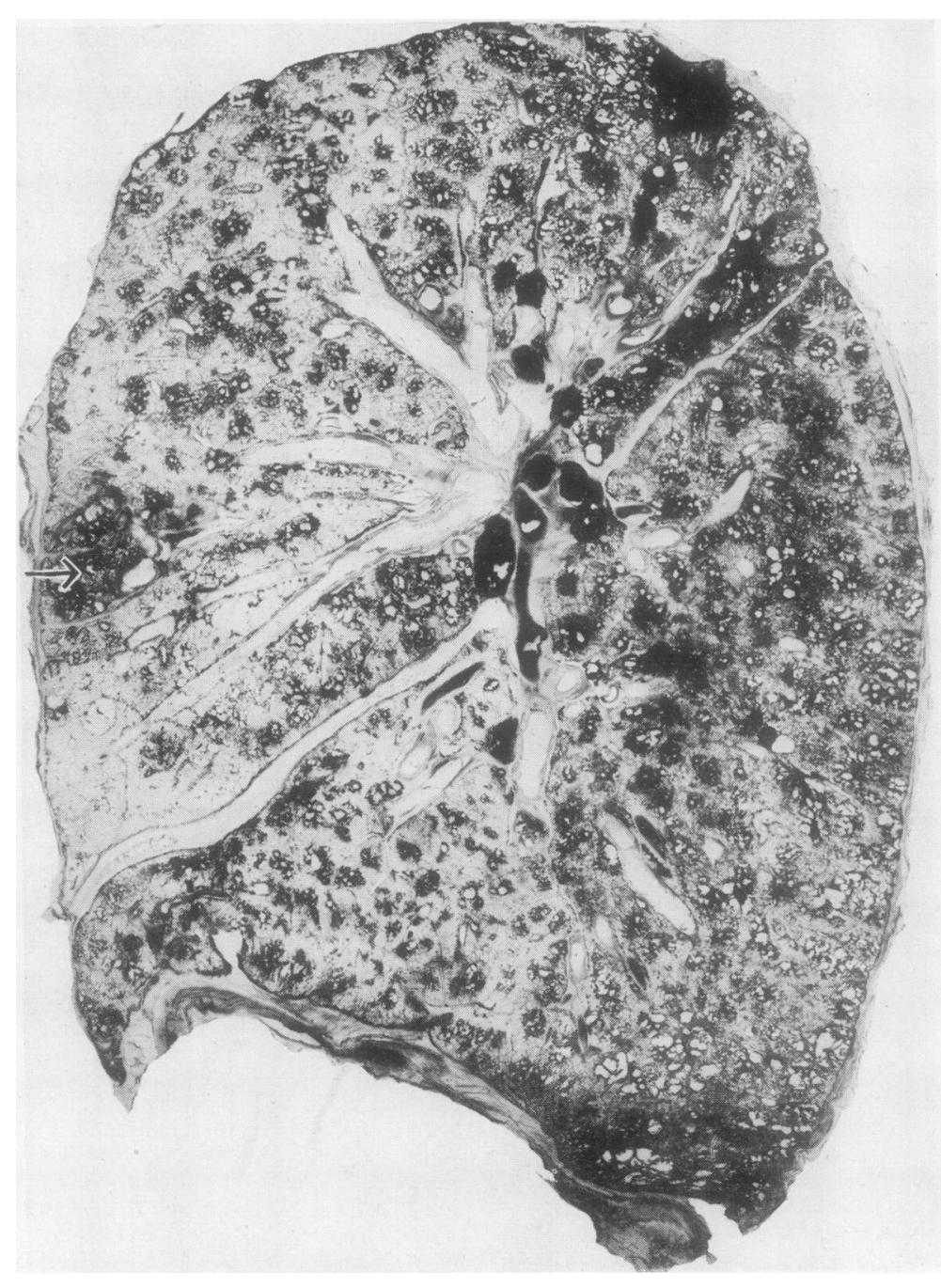


five years of his life he had been employed at a Royal Ordnance factory. He had had repeated attacks of "pleurisy, influenza, and bronchitis" over many years and became increasingly dyspnoeic during the last eight years of his life. It was on account of the dyspnoea that he ceased mining. There was no personal or family history of tuberculosis, and no history of contact with grain or birds of any sort was obtained. Repeated microscopical examination of his sputum for tubercle bacilli gave consistently negative results. Radiological examinations of the chest seven and two years before death showed the characteristic features of pneumoconiosis as found in Welsh coal workers; there was no apparent change in the radiological appearances on the second occasion and no suspicion of any condition other than pneumoconiosis. Only for the last two months of life was he compelled by dyspnoea and congestive heart failure to stop all work.

At necropsy there was no evidence of wasting. There was severe dependent oedema. Both ventricles of the heart were hypertrophied and dilated, and the liver and spleen showed chronic venous congestion. The pleurae were adherent throughout and the lungs were oedematous. In the upper lobe of each lung an area of massive fibrosis occurred and there were some

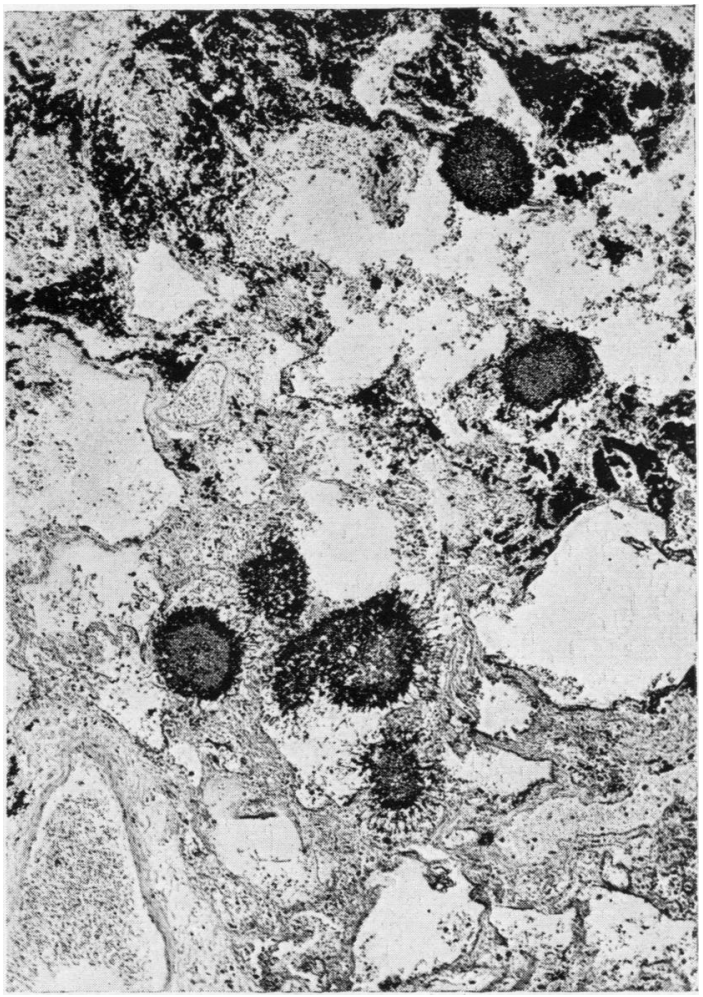

FIG. 2.-Colonies of aspergillus growing in necrotic and inflamed lung tissue. ( $\times$ 48: stain, haematoxylin and eosin.)

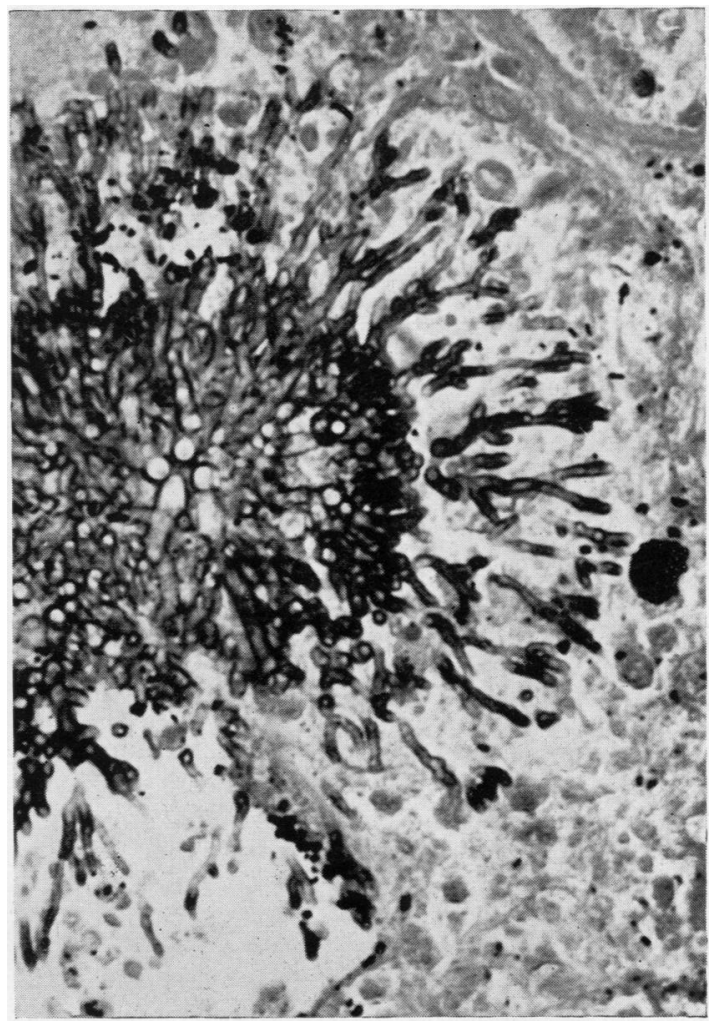

FIG. 3.-Details of a colony of the aspergillus. ( $\times 380$ : stain, haematoxylin and eosin.)

fibrous nodules nearby. The remainder of the lungs was largely occupied by many soft dust macules together with their related focal emphysema, which was of moderate severity. At the anterior margin of the right upper lobe was a greyish, infarct-like area of consolidation measuring $3 \frac{1}{2} \mathrm{~cm}$. at its widest point (Fig. 1), and in the left lower lobe there was a centrally placed consolidation with abscess formation. This abscess was transversed by many thrombosed arteries. The hilar glands were black and somewhat enlarged, but quite soft. Death was considered to be due to congestive heart failure, the result of massive and focal pneumoconiosis.

Histological examination of the lungs showed the usual features of infective and simple pneumoconiosis such as occur in coal workers (Heppleston, 1947). The infarct-like consolidation in the right upper lobe proved to be an area of suppurative pneumonia in which necrosis was severe and some haemorrhage had occurred. Fibrosis was not a prominent feature and the associated pleurisy was actually undergoing organization, suggesting that the lesion was of fairly recent origin. Intimately connected with the suppurative process were many colonies of an actively growing fungus (Figs. 2 and 3). The mycelium grew on the inner aspect of bronchiolar walls and sometimes throughout 
their thickness. In other places compact and rounded mycelial masses occurred in alveolar spaces, but in one area the fungus had invaded one of the dust macules enclosed in the area of suppuration. The mycelium was septate and branched, and was largely decolorized when stained by Gram's method. Serial sections were studied, but fructification organs were imperfectly developed throughout, as is usual when the fungus is growing in tissue. Nevertheless there was no doubt of its identity as an aspergillus, probably of the species $A$. fumigatus. The left lower lobe also showed suppurative changes, and the existence of arterial thrombosis was confirmed. Evidence of organization was detected in and around this lesion as well as in the thromboses, but, despite a general resemblance to the mycotic lesion in the right upper lobe, no trace of fungal structure could be found. The bronchi throughout were chronically inflamed and there was some patchy thickening of the intima of branches of the pulmonary arteries.

Case 2.- J.H.R., aged 56, was employed underglound in the Staffordshire coal field for 30 years, and had been totally incapacitated for work on account of pneumoconiosis during the last three years of his life.

At necropsy (performed by Dr. J. G. Dathan) the lungs showed many dust macules with related focal emphysema scattered throughout their substance. The lung bases were congested and there was an area of

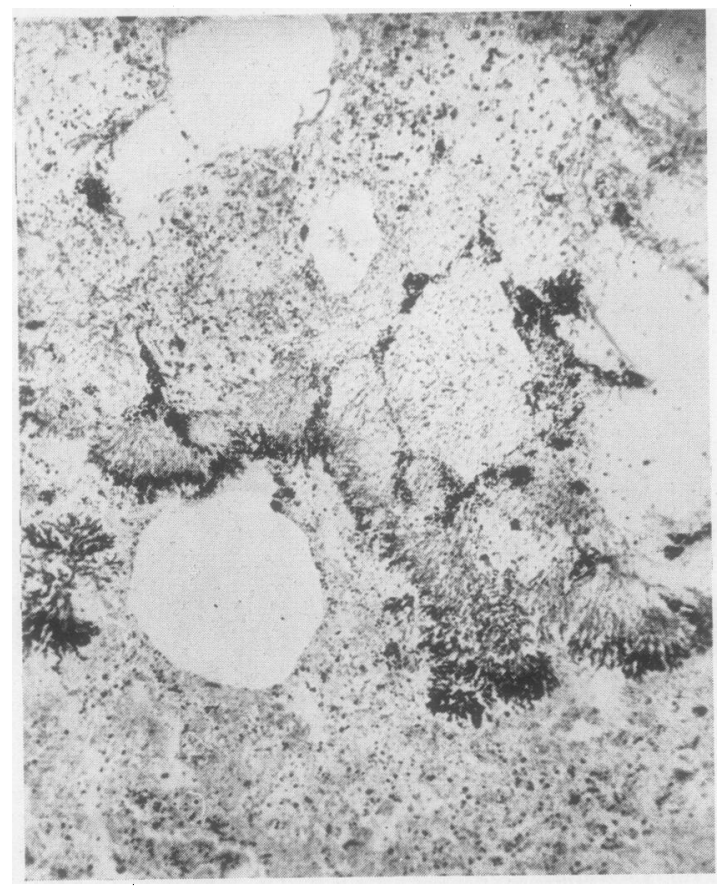

FIG. 4.-Wave-like growing edge of aspergillus in consolidated area of lung. ( $\times 70$ : stain, celestin blue.)

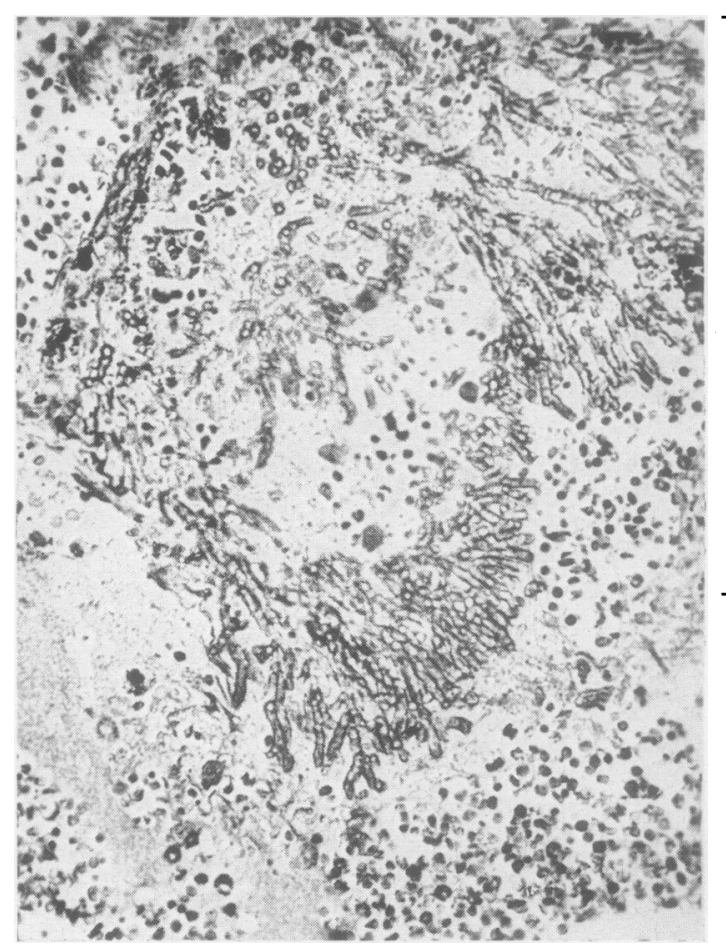

FIG. 5.-Aspergillus growing in wall of small bronchus. ( $\times$ 200: stain, celestin blue.)

consolidation on the right side. Signs of congestive heart failure were present.

An unstained paraffin section of the consolidated lung showed, macroscopically, mottled patches with ill defined margins. On microscopical examination $\dot{0}$ the lungs exhibited the usual features of simple pneu- 3 moconiosis in a coal worker together with a few small infective nodules mostly situated subpleurally. In the consolidated lung the vesicles and bronchioles were filled with degenerate erythrocytes and a little blood 옹 pigment, both free and in phagocytes. There was $D$ no inflammatory reaction, and the appearances suggested an infarction in the process of resolution rather than bronchopneumonia. The respiratory $O$ bronchioles and alveoli contained numerous fruc- $N$ tating heads of Aspergillus fumigatus (Figs. 4 and 5). N This fungus grew more profusely in the peripheral $\omega$ portions of the consolidated area and had a distinct tendency to spread in a wave-like formation. The mycelium appeared to be formed in the walls of the $\mathbb{D}$ air passages, being most obvious in bronchioles and $\stackrel{\mathcal{C}}{\rightarrow}$ alveolar ducts. Growth took place both along the $T$ inside of the bronchiolar wall and outwards into the $\bar{O}$ neighbouring alveoli. The commonest appearance $\overrightarrow{\mathbb{D}}$ was that of a row of sterigmata projecting into the $\frac{O}{\mathrm{D}}$ airway with the basal portion of the colony partially $\varrho$ concealed amongst the collagenous and elastic fibres 
of the bronchiolar wall. The fungus was not seen invading infective nodules or dust macules. The mycelium was negative to Gram's stain. The morphology of the fungus was subject to some variation. Fragments of sterigmata and detached conidia were frequently found lying free in an alveolus or alveolar duct, usually surrounded by red corpuscles. Completely intact specimens from foot cell to terminal conidia were rare. The conidiophore $(50-60 \mu)$ was non-septate and smooth-walled. The conidial heads showed the usual flask shaped vesicles $(20-25 \mu)$ with a row of closely packed sterigmata (15-20 $\mu$ ) in single series arranged over the distal half of the heads and several rows of globose conidia $(2-3 \mu)$. Ascopores were not found.

\section{Discussion}

Much of the literature on pulmonary aspergillosis is based on the identification of the fungus by culture of the sputum or on radiological evidence. Aspergilli are ubiquitous, and probably many of the species recovered from the sputum are non-pathogenic. Even when the pathogenic $A$. fumigatus is so isolated it is not always certain to be the cause of disease evident clinically and radiologically. Reliance on cultural evidence alone to establish the diagnosis may thus incur error. This is particularly the case in those instances where the aspergillosis is not the primary condition but in which there is an underlying disease process on to which the fungus infection has been grafted. Such is the sequence of events in the cases here presented, the primary condition being pneumoconiosis. Infection by an aspergillus in these two men has been established beyond doubt by post-mortem investigation. Ideally identification of the fungus in lesions should be confirmed by cultural methods, but in our cases the nature of the infection was recognized only on histological examination, by which time it was too late to adopt bacteriological procedures. Nevertheless the morphological characteristics of the fungi in the sections were sufficient to establish their identity with reasonable certainty.

The source of the aspergillus infection in these two coal workers is not known, but certain considerations are relevant. Experience of cutaneous sporotrichosis in South African gold mines led Brown, Weintroub, and Simpson (1947) to incriminate the timber used underground as the medium on which the fungus grew and from which infection occurred. No evidence is available, however, to show whether aspergilli, pathogenic or not, grow on timber in mines or whether moisture underground favours the appearance of this group of fungi, although Fawcitt (1938) records the recovery of an aspergillus from the air and the ore of a Cumberland haematite mine, and from the sputum of a coal miner (Fawcitt, 1949, personal communication). Evidence against infection from an underground source in our cases are the facts that the disease appears to be of relatively short duration whereas the men had ceased mining five and three years respectively before death. W. W. Jones (1949, personal communication) suggests that fungus infection from moist timber used as pit props is rare in British coal mines because a high rate of ventilation has to be maintained, and the timber props are usually withdrawn as the coal face advances. Furthermore we have been unable to trace any contact with birds or grain. Fawcitt (1938) originally suggested on the basis of his radiological and bacteriological experiences that fungus infection was frequently associated with dust disease, but he was unable to obtain satisfactory post-mortem confirmation. So far as we are aware our cases represent the only recorded instances in which any form of pneumoconiosis has been shown on histological grounds to be associated with infection by an aspergillus. Fawcitt does mention the recovery of Syringospora albicans from the lungs of a haematite miner, but this man had no pneumoconiosis.

An observation bearing on this problem has recently been made by one of us (A. G. H.) in the course of an experimental investigation of coal workers' pneumoconiosis. Out of a group of over 80 rabbits, exposed underground to airborne dust in one of the steam coal mines of South Wales, two have developed pulmonary aspergillosis, probably due to $A$. fumigatus. Although the fungus infection may have originated in the food or in the hay used as bedding, the other rabbits of the group, kept under identical conditions, have not developed a mycosis. Furthermore no instance of aspergillosis has been detected in numerous animals maintained in the laboratory on similar food and bedding. No animal of the group had any contact with birds, so that the only remaining possibility appears to be infection from an underground source. However, fungal growth on timber or other materials has not been observed in this particular mine. The amount of dust which has accumulated in the lungs of these two rabbits seems insufficient to act as a predisposing cause of the aspergillosis.

\section{SUMMARY}

Two cases are presented in which infection by an aspergillus, diagnosed by post-mortem examination, was associated with that form of pneumoconiosis which occurs in coal workers. The rarity 
of the association is emphasized. There was no evidence pointing to a source of infection underground.

For confirmation of the diagnoses of aspergillosis we are indebted to Dr. J. T. Duncan, of the London School of Hygiene and Tropical Medicine. Drs. R. W. Thomas and W. W. Jones kindly afforded us access to the clinical and occupational records of Cases 1 and 2 respectively, and Professor Jethro Gough gave us his advice. We are also indebted to Mr. J. P. Napper for Figs. 2 and 3, and to Mr. D. Stevenson Clark for Figs. 4 and 5.

\section{REFERENCES}

Brown, R., Weintroub, D., and Simpson, M. W. (1947). Sporotrichosis Infection on Mines of the Witwatersrand. Transvaal Chambers of Mines. Johannesburg.

Coe, G. C. (1945). Ann. intern. Med., 23, 423.

Fawcitt, R. (1938). Amer. J. Roentgenol., 39, 19.

Heppleston, A. G. (1947). J. Path. Bact., 59, 453.

Jacobson, H. P. (1932). Fungus Diseases, p. 270. Chas. C. Thomas. Springfield. Illinois.

Jones, W. W. (1949). Personal communication.

Ordstrand, H.S. van (1940). Cleveland Clin. Quart., 7,66. Thom, C., and Raper, K. B. (1945). A Manual of the Aspergilli. Baillière, Tindall and Cox. London. 